\title{
Lebensstile als Beitrag zu einer nachhaltigen Entwicklung
}

\author{
Nachhaltige Entwicklung im Alltag ist eine \\ Frage des Lebensstils. Doch wie kann man \\ mehr Menschen dazu bewegen, ihr Leben \\ nachhaltig zu gestalten? Durch die Analyse \\ von Bildungs-, Beratungs- und Erlebnis-Ange- \\ boten werden Erfolgsfaktoren für eine Verbrei- \\ tung nachhaltiger Lebensstile identifiziert. \\ Von Wilma Mert und Ulrike Seebacher
}

$\mathbf{F}_{\mathrm{d}=\mathrm{u}}^{\mathrm{u}}$ ür die industrialisierte Welt wird immer offensichtlicher, dass sie ihre bisherigen Lebens- und Wirtschaftsformen nicht mehr lange aufrechterhalten kann. Ein relevanter Teil des Ressourcenverbrauchs ist direkt oder indirekt auf die vorherrschenden Konsum- und Lebensstilmuster zurückzuführen. Viele nationale und internationale Nachhaltigkeitsstrategien und Umsetzungsprogramme setzen auf eine stärkere Verbreitung nachhaltiger Lebensstile.

Bei Lebensstilen handelt es sich nach Reusswig (1994) um relativ stabile soziokulturelle und handlungspraktische Muster, die gewählt werden können. Sie besitzen eine stark expressive Note und mit dem jeweiligen Lebensstil sind Attribute verbunden, die Menschen von anderen abgrenzen oder mit anderen verbinden. Aus Sicht der Lebensstilforschung haben Lebensstile als Triebkraft und Steuerungsmechanismus einer nachhaltigen Entwicklung nur begrenztes Potenzial. Denn neue, nachhaltige Lebensstilalternativen treffen auf bereits bestehende Lebensstilmuster, die identitätsstiftend sind.

Die Aufgabe bestehender Muster bedeutet somit auch die Aufgabe von Orientierungssicherheit und Identität. Die zu erwartenden Vorteile neuer Lebensstile müssten also eindeutig erkennbar sein, die Transaktionskosten der Umstellung mit aufwiegen sowie wiederum Sicherheit und Identität bieten können (Lange 2005).

\section{Das Forschungsprojekt NENA}

Das österreichische Bundesministerium für Wissenschaft und Forschung hat im Rahmen des Programms proVISION das Forschungsprojekt NENA - Netzwerk Nachhaltige Lebensstile beauftragt, zu untersuchen, wie durch Bündelung vorhandener Ressourcen weitreichendere Effekte bei der Änderung von Lebens- und Konsumgewohnheiten im Sinne der Nachhaltigkeit zu erreichen sind.
Ausgangspunkt war die Beobachtung, dass es zwar viele Einzelinitiativen und Akteure gibt, die sich um verstärkte Akzeptanz einer nachhaltigen Lebensweise bemühen, diese aber vielfach voneinander unabhängig operieren. Daraus ergaben sich folgende Forschungs- und Entwicklungsfragen: Wie kann man Akteure bei Ihrer Arbeit unterstützen? Wie können sie vernetzt werden? Wie kann man Kooperationen fördern?

Im Rahmen des Projektes wurden institutionelle Bildungsangebote, Kampagnen, Projekte und Informationsmaterialien analysiert, die dazu anregen nachhaltiger zu leben. Berücksichtigt wurden die Bereiche Ökologie, zum Beispiel Umweltschutz allgemein, ökologische Zusammenhänge und Mülltrennung, Ernährung, gesellschaftliches Zusammenleben, Konsum, Mobilität, Energie, Gesundheit, Bildung, Bauen und Wohnen, Freizeit und Bekleidung.

Ein Netzwerk wurde gegründet, mit dem Ziel vorhandene Ressourcen im Bereich Nachhaltige Lebensstile zu bündeln, von erfolgreichen Beispielen zu lernen, innovative Ideen zu entwickeln und, im Sinne des Ansatzes des Partizipativen Sozialen Marketings, gemeinsam mehr zu bewegen.

Eine Analyse bestehender Angebote, Interviews, Diskussionen bei Netzwerktreffen und Fokusgruppen mit Kund(inn)en dieser Angebote, sollten Aufschluss über Hemm- und Erfolgsfaktoren geben. Die Ergebnisse sind in einem Analysebericht zusammengefasst, der Empfehlungen für Optimierung und Weiterentwicklung von Angeboten enthält.

\section{Pluralistische Lebensentwürfe anerkennen}

Betrachtet man Nachhaltigkeit weniger als Inhalt, sondern vielmehr als gesellschaftlichen Veränderungsprozess, stellt sich zuallererst die Frage, wie die erfolgreiche Verbreitung nachhaltiger Lebensstile mit der bestehenden Pluralität von Lebensentwürfen zusammenpasst, wird doch damit eine Homogenisierung der Gesellschaft im Sinne der Nachhaltigkeit angestrebt.

Rückmeldungen der Teilnehmer(innen) an Fokusgruppen, Interviews und Netzwerktreffen im Rahmen des Forschungsprojektes NENA zeigten deutlich: Einen einzig richtigen nachhaltigen Lebensstil kann es nicht geben, stattdessen sind pluralistische Lebensentwürfe anzuerkennen. Bildungs-, Beratungsund Erlebnisangebote sollen die Auswirkung des eigenen Lebensstils bewusst machen. Sie sollen dazu motivieren, aus der Fülle der Möglichkeiten und innerhalb der einzelnen Bedarfsfelder wie Mobilität, Ernährung oder Gesundheit diejenigen zu wählen, die eine nachhaltige Entwicklung positiv beeinflussen. In erster Linie geht es darum Angebote zu schaffen, die sich $\rightarrow$ 
in bestehende Lebenskonzepte integrieren lassen. Tatsächlich beschrieben die Befragten einen nachhaltigen Lebensstil vielfach als aufwendig, schwierig und lustlos. Solange nachhaltige Handlungsweisen als schwierig in den Alltag integrierbar wahrgenommen werden, kann man nicht damit rechnen, dass Menschen sich freiwillig dafür entscheiden.

Obwohl das Bewusstsein für die Notwendigkeit nachhaltiger Lebensweisen durchwegs gut ausgeprägt ist, herrscht dennoch ein Informationsdefizit, wenn es um konkrete Entscheidungen geht. Oft fühlten sich die Befragten überfordert zu entscheiden, welche von zwei Alternativen die nachhaltigere ist. Ein Beispiel wäre die Wahl zwischen dem Kauf von Bioprodukten oder fair produzierten Produkten. Hier besteht der Wunsch nach klaren Anleitungen und Entscheidungshilfen. Darüber hinaus gilt es, Menschen zu motivieren, Alternativen auszuprobieren, neue Handlungsmuster zu üben und schließlich zu nachhaltigeren Gewohnheiten zu machen.

\section{Kooperationspotenziale nutzen}

Die Instituts- und Angebotslandschaft im Bereich nachhaltige Lebensstile in Österreich ist durch eine breite Vielfalt an Akteur(inn)en und Angeboten gekennzeichnet. Dadurch gibt es ein großes Potenzial, mit anderen Organisationen zu kooperieren, die in einem komplementären Kernbereich tätig sind. Solche Kooperation bietet die Chance, voneinander zu lernen und mit komplementären Zugängen und Zielgruppen gemeinsam mehr Wirkung zu entfalten. Die Chance, bei den jeweiligen Zielgruppen sowie in der Öffentlichkeit verstärkt wahrgenommen zu werden, wird durch gemeinsame Strategien und Aktivitäten gesteigert.

Angebote, die Themen des nachhaltigen Lebensstils aufgreifen, sind in allen Bundesländern Österreichs vertreten. Darüber hinaus gibt es vielfältige Anregungen aus dem deutschen Sprachraum. Eine Übertragung von erfolgreichen regionalen Konzepten in jeweils andere Bundesländer wäre ein überlegenswerter Schritt, ebenso wie die Bildung von Kooperationen innerhalb einer Region, mit einander ergänzenden Themen und Designs.

Das Zur-Verfügung-Stellen von Unterlagen, die über Literatur- und Internettipps hinaus gehen, wäre ein weiterer Schritt in Hinblick auf Vernetzung, Kooperation und gegenseitige Unterstützung. Dies setzt aber voraus, das Konkurrenzdenken nicht im Vordergrund steht.

Ein verstärktes Annehmen der Thematik nachhaltiger Lebensstile von kirchlichen Einrichtungen, Umweltschutzverbänden und Sozialvereinen wäre im Hinblick auf eine Stärkung der Breitenwirkung und Synergieeffekte höchst wünschenswert. Auch hinsichtlich der Kooperation mit Wirtschafts-, Touristikund Freizeitbetrieben gibt es ungenutzte Potenziale.

In der Erwachsenenbildung und im schulischen Bereich gibt es verhältnismäßig viele Angebote und Tools im Bereich nachhaltige Lebensstile. Lediglich für Kinder im Kindergartenalter ist noch ein Defizit feststellbar, wobei der internetbasierte Fokus der Recherche womöglich ein falsches Bild vermittelt.
Ungenutzte Potenziale sind bei folgenden Zielgruppen auszumachen:

I Menschen mit Sprach- oder Lernschwierigkeiten, Hör- oder Sehbeeinträchtigung oder anderen besonderen Bedürfnissen

- Menschen mit nicht deutschsprachigem Hintergrund, Lehrlinge und Jugendliche im außerschulischen Bereich, Senior(inn)en

I Personen, die in Land- und Forstwirtschaft tätig sind

Besonders hervorzuheben ist die Zielgruppe der Jugendlichen und Senior(inn)en. Beide Zielgruppen befinden sich in einer Lebensphase, die mit viel Orientierung beziehungsweise Neuorientierung einhergeht, wodurch oft größere Offenheit besteht, sich mit neuen Thematiken auseinanderzusetzen.

Es gibt ein eindeutiges Defizit in Bezug auf Geschlechtergerechtigkeit bei den Angeboten. Marketingexpert(inn)en raten von einer Unisex-Kommunikation ab, da Männer und Frauen unterschiedliche Bedürfnisse haben und unterschiedlich angesprochen und informiert werden wollen. Die Berücksichtigung geschlechtergerechter Kriterien in der Ansprache könnte die Durchschlagskraft der Angebote verbessern. Dabei sollte genau überlegt werden, welche Form der Vermittlung für Männer und Frauen adäquat ist.

Bei der Bewerbung von Angeboten im Bereich nachhaltige Lebensstile dominieren klassische Kommunikationsinstrumente. Netzwerke, institutionelle Partnerschaften und Kooperation sowie Vermarktung per Internet werden genutzt, um eigene Angebote $\mathrm{zu}$ verbreiten.

Teilnehmer(innen) der Fokusgruppen geben folgende Empfehlungen für die Vermarktung nachhaltiger Lebensstil-Angebote:

- Bessere Information

- Mehr Kampagnen und Werbung für Nachhaltigkeitsthemen

【 Häufige Ansprache (kurze Intervalle) in kleinem Umfang mit gut fassbaren und verständlichen Botschaften

- Kommunikation von pfiffigen, alltagstauglichen Handlungsideen und -beispielen

- Einsatz von Testimonials/Vorbildern mit Persönlichkeiten aus Gesellschaft, Sport und Politik

- Nachhaltigkeit erlebbar und sichtbar machen

- Zielgruppenspezifische, individuelle Ansprache wählen

I Individuelle Vorteile und Zusatznutzen sichtbar machen und kommunizieren

\section{Soziales Marketing für Nachhaltigkeits- kommunikation}

Soziales Marketing ist eine Strategie zur Verhaltensänderung auf freiwilliger Basis. Es basiert auf der Annahme, dass gesellschaftliche Anliegen nach denselben Mechanismen vermarktbar sind wie kommerzielle Produkte und Dienstleistungen. Tatsächlich ergaben sozialwissenschaftliche Forschungen, dass Kampagnen, die gesellschaftliche Themen aufgreifen, dann am erfolgreichsten sind, wenn sie Produktwerbekampagnen ähneln (Kotler/Roberto 1991). 
Soziales Marketing wird als sehr vielversprechend betrachtet, wenn es darum geht nachhaltige Lebensstile zu verbreiten. Sein Potenzial liegt in der starken Kundenorientierung sowie in der formalisierten Planung. Eine Besonderheit besteht darin, dass Soziales Marketing im Vergleich zum Profit-Marketing viel stärker in der Lage ist, die aktive Beteiligung von Bürger(inne)n anzuregen und bestehende soziale Netzwerke zu aktivieren (Hübner 2005).

Eine Sonderform des Sozialen Marketing, nämlich das Partizipative Soziale Marketing scheint für die Verbreitung nachhaltiger Lebensstile von besonderem Interesse (Prose 1994, Prose/Hübner 1995). Partizipatives Soziales Marketing zielt darauf $\mathrm{ab}$, einen Schneeballeffekt zu initiieren, bei dem eine Idee selbstständig Verbreitung findet. Dabei setzt man auf folgende Elemente:

I Kommunikationswege bestehender Netzwerke und den Einfluss von Meinungsführern nutzen, sowie

I Modellverhalten von Bezugspersonen als Vorbildwirkung einsetzen.

Wie sich zeigte, ist Soziales Marketing den Institutionen, die im Bereich nachhaltiger Lebensstile tätig sind, kaum ein Begriff und wird bei Planungen dementsprechend auch nicht berücksichtigt. Marketing wird eher mit groß angelegten Kampagnen in Verbindung gebracht, die entsprechende finanzielle Mittel erfordern, die gerade kleinere Institutionen oder Projekte nicht haben. Eine weitere Schwierigkeit besteht darin, dass es gerade bei kleineren Institutionen keine fixen Zuständigkeiten für Marketingfragen gibt und entsprechendes Wissen fehlt.

Viele Kampagnen beginnen oft als kleine Initiativen, die im Laufe der Zeit zu großen Bewegungen heranwachsen. Daher könnten die analysierten Angebote durch eine gezielte, strategische Planung anhand eines Marketingzyklus, wie es Soziales Marketing vorsieht, auf jeden Fall profitieren.

Der Ansatz des Partizipativen Sozialen Marketings zur Verbreitung nachhaltiger Lebensstile über bestehende soziale Netzwerke wurde bisher nur in vereinzelten Kampagnen und eher unsystematisch aufgegriffen. Nachhaltige Entwicklung benötigt aber eine breite Beteiligung, um zu funktionieren. Bisher sind Defizite hinsichtlich der systematischen Partizipation von Zielgruppen zu verorten. Viel zu oft wird in erster Linie über nachhaltige Themen kommuniziert, statt Menschen aktiv an einer nachhaltigen Entwicklung zu beteiligen und die Selbstorganisation zu fördern.

Um Partizipatives Soziales Marketing bei österreichischen Institutionen zu forcieren, wurde das Netzwerk NENA nachhaltige Lebensstile initiiert. Die bisher in der Datenbank erfassten rund 80 Institutionen wurden zur Teilnahme an zwei Netzwerktreffen und einer wissenschaftlichen Abschlussveranstaltung eingeladen. Die Netzwerktreffen mit je 20 bis 25 Teilnehmer(inne)n standen unter dem Motto Querdenken sowie Vermarktung und Vernetzung. Das Netzwerk kann als erster Impuls betrachtet werden, mit dessen Hilfe weitergehende Aktivitäten zur Etablierung nachhaltiger Lebensstile gesetzt werden sollen. Das Interesse der Teilnehmer(innen) liegt darin, dass sie ihre Stand- punkte und Strategien bezüglich der Förderung nachhaltiger Lebensstile einbringen und somit die Entwicklung des Netzwerkes inhaltlich und strukturell beeinflussen können. Im Interesse der Netzwerkpartner ist es, neues methodisches Know-how zu erwerben und ihre strategische Kompetenz durch den Erfahrungsaustausch untereinander zu erhöhen.

Das Forschungsteam übernahm im Rahmen des Projektes die Koordination des Netzwerkes und hat als Initiatorin vor der Bildung dieses Netzwerkes schon in einigen Grundsatzfragen Klärungen und Einschränkungen eigenständig vorgenommen.

Nach dem Verständnis des Teams handelt es sich bei NENA um ein an ideellen Zielen orientiertes sowie um ein wissensorientiertes Netzwerk. Bei zunehmenden Kooperationen zwischen den einzelnen Mitgliedern kann sich das Netzwerk durchaus auch zu einem gewinnorientierten Netzwerk erweitern. Gewinnorientiert ist dabei so zu verstehen, dass der gesellschaftliche Beitrag, den Netzwerkpartner wie zum Beispiel Nichtregierungsorganisationen und Bildungseinrichtungen mit ihren Aktivitäten im Themenfeld nachhaltige Lebensstile leisten, durch die Netzwerkarbeit längerfristig finanziell abgesichert werden kann.

\section{Verbreitung nachhaltiger Lebensstile}

NENA ist prinzipiell auf Dauer angelegt. Es sind unterschiedliche Aktivitäten vorstellbar. Dem offenen Netzwerkcharakter entsprechend sind weder gemeinsames Geschäftsverständnis noch Kooperationen spezifiziert. Ein einheitliches Auftreten nach außen hin ist vorstellbar, jedoch nicht notwendig und auch noch nicht geregelt. Das Netzwerk ist durchlässig, das heißt neue Partner(innen) können formlos dazu kommen, andere aussteigen. Ein Wachsen durch neue Mitglieder ist erwünscht. Bisher ist das Netzwerk nur für Organisationen offen, Privatpersonen zählen nicht zur Zielgruppe der beworbenen Mitglieder. Dies gilt auch dann, wenn sie als Lebensstilpioniere zukunftsfähige Lebensweisen bereits vorleben. Voraussetzung für die Mitgliedschaft einer Organisation ist die Orientierung am Leitbild einer nachhaltigen Entwicklung und vorhandene Aktivitäten in einem Teilbereich nachhaltiger Lebensstile. Der gemeinnützige Charakter dieser Aktivitäten ist ebenfalls wesentlich. Als Organisationen sind bisher Bildungseinrichtungen, Forschungsinstitute, Umwelt- und Unternehmensberatungen, Nichtregierungsorganisationen und Vereine vertreten.

Als Aktivitäten des Netzwerkes sind wünschenswert:

I Entwicklung neuer, innovativer Ansätze zur Förderung zukunftsfähiger Lebensweisen,

I Schaffen einer übergreifenden Marketingplattform für Angebote im Bereich nachhaltige Lebensstile,

- Erschließen neuer Märkte durch neue Angebote und die erweiterten fachlichen und personellen Ressourcen,

- Kosten- und Zeiteinsparungen durch gegenseitige Unterstützung bei Entwicklung, Bewerbung oder Durchführung von Angeboten, 
【 gemeinsame Sammlung und Auswertung von ZielgruppenInformationen,

- gemeinsame Marktentwicklung durch neue Finanzierungsmöglichkeiten und komplementäre Zugänge.

Das Eingehen institutioneller Kooperationen innerhalb des Netzwerkes NENA könnte nach innen eine Vielzahl von Vorteilen bringen. Dazu gehören die Konzentration auf die jeweiligen Stärken der einzelnen Mitglieder, Wissenszuwachs und Innnovation, Nutzen von Vorteilen aufgrund der gemeinsamen Größe und die dadurch höhere Flexibilität und konstantere Leistungsfähigkeit, gegenseitige Aufträge, Erweiterung des Akquisitionsfeldes und Verbesserung der Auftragsqualität.

Nach außen hin könnte das Sichtbarwerden als ein Großes statt vieler kleiner institutioneller Akteure den Marktauftritt und die Wahrnehmung des Themas zukunftsfähige Lebensstile verbessern.

\section{Schlussfolgerungen und Ausblick}

Viele der von den Interviewpartner(inne)n und Teilnehmer(inne)n der Netzwerktreffen genannten Faktoren für eine erfolgreiche Verbreitung nachhaltiger Lebensstile sprechen dafür, das Anliegen in einem Netzwerk gemeinsam weiter zu verfolgen.

Die Ergebnisse des Projektes NENA in Form von Analysebericht, Datenbank und Netzwerk sind ein Beitrag, um Angebote und Werkzeuge zu verbessern und neue Kooperationen zu initiieren. Zugleich verhelfen sie der Thematik nachhaltiger Lebensstile zu mehr Sichtbarkeit, Akzeptanz und Breitenwirkung.

Für die Weiterentwicklung sowie für die Neukonzeption von Angeboten und Werkzeugen stehen durch den Analysebericht und die in einer Datenbank gesammelten Beispiele vielfältige und konkrete Anregungen zur Verfügung (1). Darüber hinaus geben fünf Leitfäden Hilfestellung und Anregungen für die
Themenfelder Ideenfindung, Partizipation, Marketing und Geschlechtergerechtigkeit und Evaluierung, die als bisherige Schwachpunkte identifiziert wurden.

\section{Anmerkungen}

(1) Der Analysebericht sowie die Datenbank mit Beispielen stehen auf www.nenanet.at zur Verfügung.

\section{Literatur}

Hübner, G.: Soziales Marketing. In: Michelsen, G. / Godemann, J. (Hrsg.): Handbuch Nachhaltigkeitskommunikation. Grundlagen und Praxis. München 2005. S. 287-296.

Kotler, P. / Roberto, E.: Social Marketing. Düsseldorf, Wien, New York 1991. Lange, H.: Lebensstile - Der sanfte Weg zu mehr Nachhaltigkeit? In: Michel sen, G. / Godemann, J. (Hrsg.): Handbuch Nachhaltigkeitskommunikation. Grundlagen und Praxis. München 2005. S. 160-172.

Prose, F.: Systematik von Energiespar-Kampagnen am Beispiel der Klimaschutzaktion Nordlicht. In: Senatsverwaltung für Stadtentwicklung und Umweltschutz Berlin (Hrsg.): Neue Wege im Energiesparmarketing: Materialien zur Energiepolitik in Berlin. Berlin 1994. S. 69-72.

Prose, F. / Hübner, G.: Soziales Marketing für den Klimaschutz. In: Altner, G. / Mettler-Meibom, B. / Simonis, U. / Weizäcker, E.U. (Hrsg.): Jahrbuch Ökologie 1995. München 1995. S. 285-290.

Reusswig, F.: Lebensstile und Ökologie. Gesellschaftliche Pluralisierung und alltagsökologische Entwicklung unter besonderer Berücksichtigung des Energiebereichs. Frankfurt a. M. 1994.

\section{AUTORINNEN + KONTAKT}

Dr. Ulrike Seebacher leitet den Forschungsbereich Ökologische Produktpolitik am Interuniversitären Forschungszentrum Graz und Wilma Mert arbeitet dort zu den Themenbereichen Energie, Klima, Nachhaltigkeitskommunikation und Marketing.

Interuniversitäres Forschungszentrum für Technik, Arbeit und Kultur (IFZ), Schlögelgasse 2, $8010 \mathrm{Graz}$ Österreich. E-Mail: seebacher@ifz.tugraz.at; mert@ifz.tugraz.at
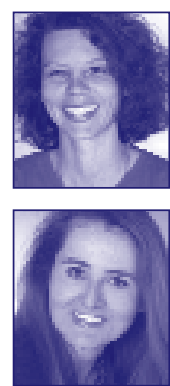

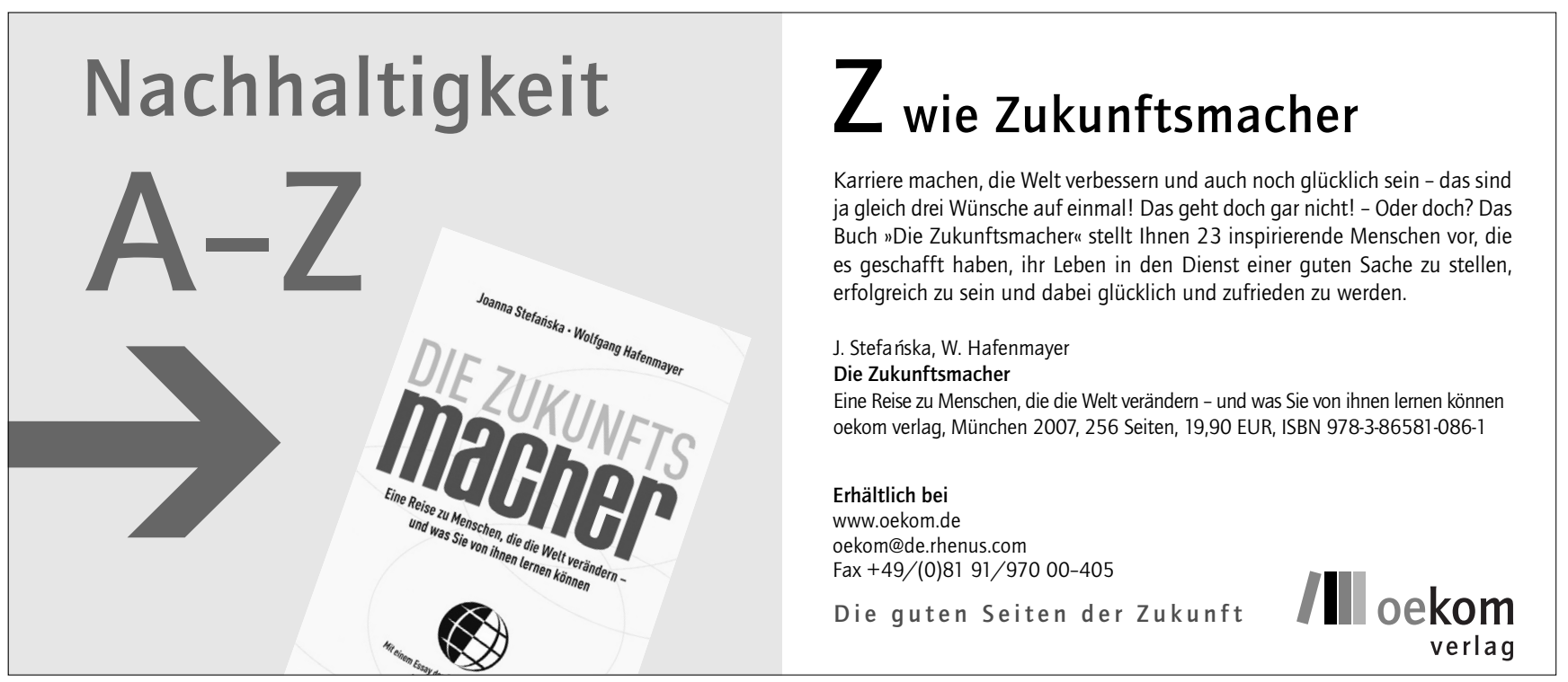


(c) 20I0 Authors; licensee IÖW and oekom verlag. This is an article distributed under the terms of the Creative Commons Attribution Non-Commercial No Derivates License (http://creativecommons.org/licenses/by-nc-nd/3.o/), which permits unrestricted use, distribution, and reproduction in any medium, provided the original work is properly cited. 